\title{
Notes hagiographiques sur l'époque du second iconoclasme
}

\author{
Tatiana A. Sénina (nonne Kassia) \\ Université d'État de l'instrumentation aérospatiale, Saint Petersburg, Russia \\ mon.kassia@gmail.com
}

$1 \quad$ La date de la mort de Saint Procope le Décapolite

La Vie de Saint Procope le Décapolite (BHG 1583), ${ }^{1}$ très courte et pauvre en renseignements historiques, ne nous donne aucune information sur la date de sa mort. L'indication « Death Year: 826 » de Dumbarton Oaks Hagiography Data$b_{a s e}{ }^{2}$ se base certainement sur la version de l'éditeur de la Vie du saint. En effet, S. Efthymiadis a identifié Saint Basile, compagnon de Procope,$^{3}$ comme un des destinataires de Saint Théodore le Stoudite. ${ }^{4}$ Cinq lettres de Théodore à l'hégoumène Basile sont conservées : elles datent de 816-818 (deux), d'après 821 (une) et de $824-826$ (deux). ${ }^{5}$ A.P. Dobroklonsky ${ }^{6}$ et, après lui, S. Efthymiadis ont considéré que toutes ces lettres seraient adressées à la même personne et que Basile aurait été d'abord défenseur ferme des icônes, mais aurait ensuite accepté un compromis avec les dogmes iconoclastes, après quoi il se serait repenti et aurait accepté une pénitence de Saint Théodore. Cependant, G. Fatouros pense que les lettres de 816-818 sont adressées à une personne et le reste

1 S. Efthymiadis, «La Vie inédite de S. Procope le Décapolite ( $B H G$ 1583) », AB, 108 (1990), pp. 313-319.

2 <http://www.doaks.org/research/byzantine/resources/hagiography-database>.

3 Il est mentionné dans la note de synaxaire sur les deux saints comme compagnon et maître de Procope (Synaxarium Ecclesiae Constantinopolitanae e codice Sirmondiano nunc Berolinensi adiectis Synaxariis selectis, ed. H. Delehaye, Bruxellis, 1902, col. 491/492.58), bien que la Vie de celui-ci ne parle pas de Basil.

4 Efthymiadis, « La Vie inédite de S. Procope », pp. 307-309.

5 Theodori Studitae Epistulae, rec. G. Fatouros, vol. 1-2 (csHв), Berlin - New York, 1992, Ep. 317 и $389,60,494$ et 495 conformément.

6 А.П. Доброклонский, Преп. Феодор, исповедник и игумен Студийский, vol. 2, Odessa, 1914, pp. $405^{-406 .}$ 
à une autre. ${ }^{7}$ À ce qu'il paraît la dernière hypothèse est plus proche de la vérité. En effet, dans la première des lettres à Basile Théodore dit :

«En ayant appris tout ce que ta sainteté a supporté avec ton saint novice, j'ai beaucoup glorifié notre bon Dieu pour ce que tu aies également brillé comme un foyer de lumière dans le monde ${ }^{8}$ avec les autres lumières dans cette bataille nocturne avec l'hérésie, instrument de choix, ${ }^{9}$ colonne et support de la vérité10 ! Comme vous deux êtes merveilleux, comme vous êtes agréables pour le Christ, en ayant supporté pour Lui les coups, les exils, les incarcérations, et celà jusqu'au présent !<...> Tu dis que tu n'as jamais vu l'empereur ni t'es présenté devant les chefs, et c'est pourquoi tu crains un peu. Ne crains rien, père, t'étant revêti du Christ ! Que (l'empereur) te craigne lui-même, de la même façon que les démons. Ouvre tes lèvres devant lui. La parole te sera donnée comme le Christ l'a promis ${ }^{11} \gg .^{12}$

Dans la lettre suivante Théodore, en répondant à la question de Basile sur l'attitude envers ceux qui ont commis certains péchés mais sont devenus confesseurs pendant les persécutions, ne mentionne la confession du destinataire qu'en y faisant allusion : « prie que j'approche de ton lot et ton état ». La lettre est finie par la phrase : «Salue bien mon frère Procope.$^{13}$

On peut conclure qu'au moment de la rédaction de la première lettre Basile avec son novice avaient été emprisonnés, avaient subi la flagellation et avaient été exilés, et s'apprêtaient à comparaître devant les autorités ou l'empereur luimême. Cette situation ressemble à ce qui est raconté dans la Vie de Procope, selon les données des Synaxaires et à ce que l'on apprend d'autres sources : les iconophiles avaient d'abord été exilés, mais très vite, à l'hiver $815 / 816$, rappelés à Constantinople où ils ont été emprisonnés et mal traités, après quoi les iconoclastes leur ont offert de communier une seule fois avec le patriarche iconoclaste Théodote et puis retourner à leurs monastères; cette ruse a fait tomber beaucoup de confesseurs des icônes. ${ }^{14}$ De la deuxième lettre de Theodore rédigée plus tard, nous pouvons conclure que Basile aurait supporté avec succès la rencontre avec les autorités de la capitale et n'aurait pas renoncé à la

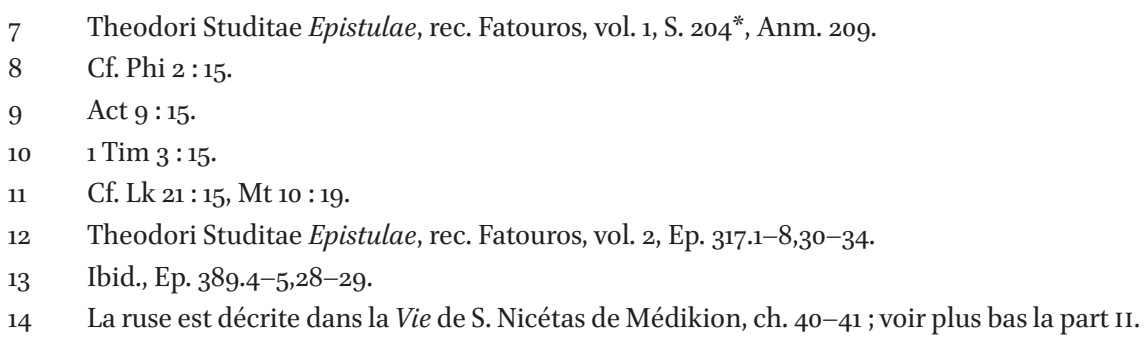


vénération des icônes ; au moment de la rédaction de la lettre, il était probablement en exil.

Quant à Basile, destinataire des trois autres lettres, il ne s'est pas montré très ferme pendant les persécutions, ni sous Léon l'Arménien ni plus tard $;^{15}$ Procope n'est pas mentionné dans ces lettres. On peût donc partager l'opinion de G. Fatouros que «l'iconophile ardent» Basile des deux premières lettres ne doit pas être identifié au destinataire pusillanime des trois autres.

S. Efthymiadis a identifié le novice Basile avec le moine Procope, destinataire d'une autre lettre de Théodore le Stoudite. ${ }^{16}$ Celà me paraît pourtant peu probable. Premièrement, dans la lettre il n'y a rien pour identifier le destinataire comme le compagnon de Basile. Deuxièmement, le contenu de la lettre ne correspond pas aux données de la Vie de S. Procope, car avant la tonsure le destinataire de Théodore était marié et avait des enfants qui étaient en vie au moment de la rédaction de la lettre et avec qui Procope habitait sous le règne de Michel II, évidemment pour éviter la communion avec les iconoclastes en restant au monastère. ${ }^{17}$ Efthymiadis pense que Procope le Décapolite est la même personne que «le frère Procope » mort en exil et mentionné dans une lettre de Théodore le Stoudite, ${ }^{18}$ mais celà est aussi improbable : dans cette lettre l'hégoumène s'adresse à sa confrérie et parle des stoudites morts, mais Procope n'était pas un moine du Stoudion.

Ainsi, il n'y a pas de bonnes raisons pour dater la mort de Saint Procope de la période entre 824 et 826 . À en croire sa Vie, il est mort en exil, et dans ce cas celà a eu lieu, compte tenu de la datation approximative des lettres de Théodore à Basile, en 818-820.

15 Ce Basil a accepté un compromis avec les iconoclastes, c'est pourquoi Théodore le Stoudite lui a imposé une pénitence et l'encourageait à renoncer à son higouménat. Voir en détails : Efthymiadis, « La Vie inédite de S. Procope », pp. 308-309.

16 Theodori Studitae Epistulae, rec. Fatouros, vol. 1, Ep. 69. Fatouros estime cette identification comme probable (Ibid., S. 210*, Anm. 227).

17 Fatouros date cette lettre de la période postérieure à $817-818$; Dobroklonskiy propose la date après 821 , ce qui est plus vraisemblable (voir : Доброклонский, Преп. Феодор, vol. 2, pp. 470-471).

18 Theodori Studitae Epistulae, rec. Fatouros, vol. 2, Ep. 503. Fatouros date cette lettre de $824-826$. 


\section{Le rôle du moine Arsène dans le retour de Saint Nicétas de Médikion à l'orthodoxie}

La Vie de Saint Nicétas de Médikion (BHG 1341) $)^{19}$ est intéressante avant tout par la description des événements de l'époque du second iconoclasme et notamment des ruses à l'aide desquelles les iconoclastes séduisaient les iconodules.

En printemps de 815 , après le concile iconoclaste, les iconodules ont subi la persécution. S. Nicétas a passé quelques mois dans une prison à Constantinople et ensuite, probablement au début de janvier de 816, il a été exilé en Asie Mineure - mais pour peu de temps. Seleument quelques jours plus tard, l'empereur a ordonné de retourner les confesseurs des icônes dans la capitale. ${ }^{20}$ Après Pâques Nicétas et les autres hégumènes orthodoxes sont tombés dans les mains de Jéan le Grammairien et se sont trouvés dans une réclusion sévère. Quelque temps passé, on leur a proposé de communier une seule fois avec le patriarche iconoclaste et retourner librement dans leur monastères, où ils pourraient professer la foi comme ils le voulaient. Cette ruse a eu l'effet que les iconoclastes attendaient, et beaucoup d'iconodules, y compris S. Nicétas de Médikion, ont consenti à prendre l'eucharistie des mains du patriarche Théodote. ${ }^{21}$ L'hagiographe tâche de disculper le saint en disant qu'il a cédé non par peur des tourments mais seulement «s'étaint laissé convaincre par les exhortations des pères », par respect pour les autres hégumènes qui avaient cédé aux iconoclastes avant lui. D’après sa Vie, Nicétas s'est repenti presque aussitôt, s'est joint de nouveau aux iconodules et pour celà on l'a arrêté et exilé de nouveau.

S. Nicétas est le destinataire de plusieures lettres de S. Théodore le Stoudite. $^{22}$ Dans une lettre à S. Naucrace le Stoudite du début de 816 Théodore chante les louanges de Nicétas pour la «bonne réponse » (évidemment pendant l'interrogatoire chez les iconoclastes). ${ }^{23}$ En été ou en automne 816 dans une lettre à l'archévêque Joseph de Thessalonique, son frère Théodore lui ap-

19 Acta Sanctorum, Aprilis, t.I, [Appendix] p. XVIII-XXVII. Dans cet article je m'oriente à la traduction russe faite par D. Afinogenov qui a utilisé le texte critique que prof. J.O. Rosenqvist lui a accordé et la version slavonne de la Vie: Житие преп. Константина из Иудеев. Житие св. исповедника Никиты, пер., сост., статья Д.Е. Афиногенова, Moscou, 2001, pp. 97-146 (la traduction slavonne est précieuse surtout parce qu'elle est faite d'après un texte grec intact ce qui permet de rectifier le texte de Acta Sanctorum).

$20 \quad$ La Vie de S. Nicétas, ch. 39.

21 Ibid., ch. 40-41.

22 Il est aussi mentionné dans quelques autres. La liste de toutes ces lettres d'après l'édition de G. Fatouros voir : PmbZ, № 5443 .

23 Theodori Studitae Epistulae, rec. Fatouros, vol. 2, Ep. 136.9-10. 
prend que l'hégumène de Médikion avec les autres « ont été entraînés sous prétexte soi-disant d'économie », par l'économe Joseph, à la communion avec les iconoclastes. Théodore nomme tous ces hégumènes « la vieille garde (xov$\sigma \tau 0 \delta^{\prime}(\alpha)$ », en sous-entendant que c'étaient les mêmes hommes qui n'avaient pas protesté contre le deuxième mariage de l'empereur Constantin vi avec Théodote par Joseph cité. ${ }^{24}$ On peut en conclure que Nicétas se tenait à l'écart de la protestation des Stoudites et leurs partisans pendant le « schisme mœchien ».

Dans quelques autres lettres à différentes personnes Théodore dit que Nicétas « a bien renoué la lutte », ${ }^{25}$ « s'est relevé après la chute » ${ }^{26}$ et a donné aux autres «le grand exemple du repentir $» .{ }^{27}$ Le Stoudite a écrit aussi à Nicétas lui-même en le louant de son repentir :

« $[\mathrm{Tu}]$ as montré le repentir plus éclatant que la défaite, tu es monté à la hauteur de la confession du Christ, tu as éclairé le monde par les rayons de la vérité. Ce sont les actions de ta sainteté paternelle qui ont réjoui les orthodoxes et frappé les adversaires d'un coup de l'arme qu'ils considéraient comme la leur. Car celui qui tient ferme ne frappe pas aussi fort que celui qui se relève après la chute par la puissance du Seigneur ${ }^{28}$

La lettre suivante de Théodore est semblable dans le contenu (Nicétas n'a peutêtre pas reçu la première ?), mais le Stoudite y écrit, en dehors des louanges, que l'économe Joseph qui avait entrainé les autres vers la chute est tombé luimême à cause de son habitude de l'« économie » auparavant. ${ }^{29}$

Ainsi, les lettres de S. Théodore confirment les renseignements de la Vie de S. Nicétas. Mais elles contiennent aussi une information absente dans la Vie. Celle-ci dit qu'après la chute, Nicétas ne soit revenu vers l'orthodoxie que de sa propre initiative. ${ }^{30}$ Mais $S$. Théodore nous fait savoir que c'était un élève de Nicétas, le moine Arsène, qui l'aurait incité à renouveler la lutte pour l'iconophilie. Le Stoudite lui envoie ses salutations à la fin de la lettre citée plus haut et ensuite il lui écrit personnellement :

$\begin{array}{ll}24 & \text { Ibid., Ep. 222.4-14. } \\ 25 & \text { Ibid., Ep. 177.38-39. } \\ 26 & \text { Ibid., Ep. 197.21. } \\ 27 & \text { Ibid., Ep. } 267.25^{-28} . \\ 28 & \text { Ibid., Ep. 255.8-15. } \\ 29 & \text { Ibid., Ep. 280. } \\ 30 & \text { Voir ch. 41-42. }\end{array}$


« Ayant reçu la lettre de ton amour et l'ayant compris, j'ai gémi beaucoup à cause du malheur advenu et surtout de la chute de ceux qui passaient pour les hommes d'élite. Ton père et mon frère unanime ont échappé à cette chute $!<\ldots>$ Je te loue surtout, frère, de tes soins pour ton précepteur. Car tes paroles de persuasion cordiales et ardentes sont vraiment les signes de l'affection d'un élève véritable et les lamentations d'un enfant sincère. La rencontre avec l'hérésie luttant contre le Christ et même une seule fois de la communion avec elle c'est la mort de l'âme. <...> Et toi qui a pleuré, qui a saisi ton père par les deux mains et l'as fait revenir à la confession précédente, tu es bienheureux $! »^{31}$

Cette lettre montre qu'Arsène a joué un rôle dans la conversion de l'hégumène de Médikion. De plus, Arsène se trouvait évidemment à côté de Nicétas avant sa chute comme après son repentir. Ils étaient ensemble au moins jusqu'à la mort de Léon l'Arménien, ce qui apparaît dans la lettre suivante de Théodore le Stoudite. ${ }^{32}$ Pourtant la Vie ne dit pas un seul mot sur cet élève de Nicétas. Plus encore, l'hagiographe dit qu'avant la chute de Nicétas c'était le jeune moine Théoctiste qui le servait et qui était emprisonné avec lui ; c'est Théoctiste qui deviendra l'hégumène de Médikion après Nicétas. ${ }^{33}$ Nous pouvons ainsi conclure que la Vie de S. Nicétas est flattante pour l'hégumène Théoctiste sous lequel elle a été écrite, et «l'honnêteté et la franchise de son auteur » qui ravissent D. Afinogenov ${ }^{34}$ étaient quelque peu limitées : la chute et le repentir de l'héros sont montrés - ce qui est compréhensible car les contemporains considéraient une telle conduite comme louable, comme en témoignent les lettres de Théodore le Stoudite (en effet, bien de personnes étaient alors tombées et ne s'en étaient point repenties), - mais l'hagiographe a passé sous silence que c'était l'élève de Nicétas qui l'avait incité au repentir.

Enfin, il est intéressant d'examiner la dernière et la plus longue des lettres de Théodore à l'hégumène de Médikion écrite sous Michel II. ${ }^{35}$ Théodore reproche à Nicétas d'approuver la profession de foi d'un certain Maximin qui est revenu à l'orthodoxie après la communion chez les iconoclastes. Ce Maximin disait qu'il «avait communié mais n'était pas en relation» avec les hérétiques. L'hégumène de Stoudion prouve, au contraire, que communier c'est

Theodori Studitae Epistulae, rec. Fatouros, vol. 2, Ep. 281.2-11,25-27.

32 Voir ibid., Ep. 422 (du début de 821), à la fin de laqelle Théodore salue les moins étant avec Nicétas « et surtout le saint Arsène ».

33 Voir ch. 40.

34 Voir :Житие ... св. исповедника Никиты, пер. Афиногенова, с. 95.

35 Theodori Studitae Epistulae, rec. Fatouros, vol. 2, Ep. $45^{2}$. 
précisément être en relation et que Maximin doit se repentir de telles opinions puisqu'elles ne sont pas orthodoxes. Si Nicétas approvait vraiment des vues pareilles, cela montre que même après avoir repenti de sa chute il ne comprenait toujours pas entièrement la profondeur de l'abîme entre les confesseurs des icônes et ceux qui préféraient éviter les persécutions, en entrant en relation avec les iconoclastes sous tel ou tel prétexte. On peut alors mieux comprendre le rôle du moine Arsène dans le repentir de S. Nicétas.

\section{La date du Panégyrique de Saint Théophane le Confesseur par Saint Théodore le Stoudite (}

Saint Théophane, l'hégumène du monastère de Megas Agros à Sigriane, exilé par l'empereur Léon V l'Arménien à l'île de Samothrace, y est mort le 12 mars 818. Les persécutions contre les iconodules ayant pris fin sous Michel II, les moines de Sigriane ont voulu transporter les reliques de leur père au monastère. D'après la Vie de S. Théophane écrite pas S. patriarche Méthode, ${ }^{36}$ cette translation a eu lieu au printemps de 821. Cette année-là, vers Pâques, la dépouille du saint a été transportée par mer au proasteion d'Hiéreia au bord de la Propontide où les moines l'ont déposée dans le martyrion de S. Procope situé à 12 milles du monastère de Megas Agros. Mais la translation finale a été retardée à cause du grand nombre de fidèles qui venaient pour vénérer les reliques. La dépouille de $\mathrm{S}$. Théophane est restée au proasteion pendant tout un an et n'a été transportée à Megas Agros qu'après la fête de Pâques suivante, c'est-àdire après le 13 avril de 822 .

Saint Théodore le Stoudite a assisté à la translation et a prononcé un panégyrique en l'honneur de S. Théophane. S. Efthymiadis qui a édité ce Panégyrique $^{37}$ l'a daté du printemps de 822 : «... à la suite de son rappel d'exil de

36 L'édition de V.V. Latichev, «Мефодия, патриарха Константинопольского, Житие преп. Феофана Исповедника. По московской рукописи № 159», Записки Российской Академии Наук, vІІІ серия, по историко-филологическому отделению, XIII.4 (1918). Sur la translation des reliques voir ch. $57-58$ de cette Vie.

37 S. Efthymiadis, «Le Panégyrique de S. Théophane le Confesseur par S. Théodore Stoudite (вHG 1792b). Édition critique du texte intégral », $A B, 111$ (1993), pp. 259-290. Il existe deux traductions commentées russes : par D. Afinogenov, «Его же (то есть Феодора, игумена Студийского) слово о перенесении из Самофракии в Сигриану священных мощей святейшего чудотворца отца нашего Феофана», dans : Преподобный Феодор Студит, Творения, т. 3: Письма. Творения Гимнографические. Эпиграммь. Слова (Полное собрание творений святых отцов Церкви и церковных писателей в русском переводе, 7), Moscou, 2012, pp. 908-918; par moi, «Феодора, игумена Студийского, Слово на перенесение с Самофраки на Сигрианскую (гору) святых останков преподоб- 
Smirne, à l'époque de la révolte de Thomas le Slave, l'abbé Stoudite résida pendant un an (821-822) au port de Kreskentios sur le golfe de Nicomédie. Par conséquent, il n'est pas impossible qu'il ait poussé une pointe jusqu'à Agros pour prononcer ce discours $\gg .38$

Mais c'est une faute manifeste. Efthymiadis invoque les Vies $B$ et $C$ de S. Théodore qui cependant disent que celui-ci a habité à Kreskentios peu de temps et après, par crainte de la révolte de Thomas le Slave, est passé à Constantinople et y est resté jusqu'à la fin de la rébellion comme plusieurs autres iconodules. ${ }^{39}$ Or, les armées de Thomas se sont empareés de toute l'Asie au cours de 821 et ont assiégé Constantinople en décembre de cette même année. L'assaut de la Ville n'a pas réussi, et Thomas a levé le siège pour quelque temps, mais l'a recommencé au printemps de 822 , et la Ville a été assiégée pendant tout un an. Naturellement les iconodules ont du se trouver à Constantinople avant le début du premier siège, d'autant plus que l'empereur Michel II, de crainte que les iconodules ne soutiennent Thomas, leur avait ordonné d'entrer dans la Ville et celà s'était passé précisément avant décembre de 821 .

Mais si S. Théodore avait habité à Constantinople depuis le décembre de 821, il n'aurait pas pu assister à la translation des reliques de $\mathrm{S}$. Théophane d'Hiéreia à Megas Agros en avril de 822. Comment résoudre cette contradiction ? S. Méthode pouvait avoir des informations inexactes, et en réalité le corps de Théophane n'était point resté dans l'église d'Hiéreia pendant un an mais avait été transporté tout de suite à Megas Agros - d'autant plus que des milliers d'hommes venant constamment au monastère pour vénérer la dépouille ${ }^{40}$ correspondent mal aux circonstances de la révolte de Thomas et de la guerre civile. L'autre explication est que S. Théodore le Stoudite n'aurait assisté qu'à la première partie de la translation des reliques, c'est-à-dire pendant leur accueil à Hiéreia, et c'était alors qu'il a prononcé son discours. Dans tous les cas son Panégyrique doit être daté de la fin du mars de 821.

нейшего и чудоносного отца нашего Феофана», dans :Жития византийских святьх эпохи иконоборчества, т. I, общ. ред. Т.А. Сениной (монахини Кассии) (Byzantina), Saint Pétersbourg, 2015, pp. 209-226.

38 Efthymiadis, «Le Panégyrique », p. 260.

39 Voir: P. Lemerle, «Thomas le Slave», Travaux et Mémoires, 1 (1965), pp. 262-263; А.П. Доброклонский, Преп. Феодор, исповедник и игумен Студийский, vol. 1, Odessa, 1913, pp. 856-862, 869-871; Th. Pratsch, Theodoros Studites (759-826) - zwischen Dogma und Pragma. Der Abt des Studiosklosters in Konstantinopel im Spannungsfeld von Patriarch, Kaiser und eigenem Anspruch, Frankfurt am Main etc, 1998, ss. 273-275. R. Cholij, Theodore the Stoudite: the Ordering of Holiness, Oxford - New York, 2002, p. 62, affirme que Michel II a fait Théodore et les autres iconodules venir à Constantinople au printemps de 822 en invoquant la Vie de Théodore où il n'y a point de données précises pour cette date. D’après Méthode : voir la Vie de S. Théophane, ch. 58. 